\section{World institute for astronomy}

SiR-We would like to offer some additional thoughts on a recent proposal for a world institute for astronomy ${ }^{1}$. As a consequence of President George Bush's speech in July 1989, the creation of a permanent scientific base on the Moon is being considered. The lunar surface is one of the best possible sites for an astronomical observatory operating across the entire electromagnetic spectrum, and indeed among the prospects being considered by NASA (the National Aeronautics and Space Administration) are a large (16metre) optical telescope and an optical interferometer ${ }^{2}$.

It is generally accepted that optical interferometry will be a tool of great astronomical utility in the next century. Several significant Earth-based projects are under way: the European Very Large Telescope, the Optical Very Large Array and a number of ambitious proposals in the United States. Space platforms are also being considered. But in the long term, the lunar surface offers a number of substantial advantages - no atmospheric hindrance at any wavelength, mechanical stability, kilometre-length baselines and an accurately known position.

The establishment of a lunar base, requiring financial and technical resources and complex management, will undoubtedly require an international effort. We suggest that in parallel with this effort, a lunar optical interferometer should be the first astronomical instrument conceived and constructed through worldwide cooperation. The creativity of individual scientists, a necessity for scientific progress, could be stimulated by such a programme, especially in developing countries where a great deal of talent remains unrevealed ("Ces Mozarts qu'on assassine") because of the lack of opportunities and stimulus.

More symbolically, an international scientific venture like this, visible from everywhere on the Earth, would demonstrate a worldwide unity in our quest to understand the Universe. It might be the twentyfirst-century equivalent of the rapid dissemination of Galileo's refracting telescope throughout Europe after the discoveries of 1610 .

Such a venture would not necessarily demand a world institute of the type proposed by Bahcall et al.'. A delocalized effort, facilitated by rapid communication links and electronic mail, could also make possible a truly worldwide, goal-orientated programme. This 'institute without walls' would naturally deal with the space agencies handling the lunar base itself.

Ground-based interferometers of the type now being developed through individual efforts around the world might also be supported initially by the world institute. If these ideas meet with interest in the scientific community, the time may be appropriate to hold joint meetings on the 'Lunar Optical Infrared Synthesis Array', and on the possibility of worldwide cooperations towards its construction. \section{National de la Recherche Scientifique,}

Université Paris VII, Observatoire de Paris, 5 Place Jules Janssen, 92195 Meudon Cedex, France.

1. Bahcail, J.N., Giacconi, R., Oda, M., Rees, M., Sagdeev, R. \& Sunyhev, R. Nature 339, 574 (1989).

2. "Lunar Optical and Infra-red Synthesis Array", workshop proc., Albuquerque, 1989 (in press).

\section{SSC poor value}

SIR - The US Secretary of Energy, James Watkins, has convened a 'blue ribbon' panel of 15 'eminent' physicists to advise him on the Superconducting Super Collider (SSC), because he feels that he can count on them to support a decision to proceed with the project, despite numerous design problems and an increase in cost to $\$ 7,000$ million. He should have convened a panel of 15 eminent poultry farmers, as they would have had the common sense to avoid two classic errors (1) "Don't put all of your eggs in one basket" and (2) "Don't count your chickens before they hatch", particularly if you have put all your eggs in one basket. US high-energy physicists would obviously like to put all of their 'eggs' in the SCC basket, and they are already counting their unhatched Higgs bosons and their airline tickets to Stockholm.

The situation at the SSC is even more precarious than most people imagine because of the sheer size of the project and the elementary laws of probability. The machine will have nearly 10,000 superconducting magnets. If even one of the magnets is not working properly, the machine will not reach its design energy. Thus, even if each individual magnet is 99.99 per cent reliable, the machine as a whole would have a reliability of $(0.9999)^{10,000}=37$ per cent. If each magnet were only 99 per cent reliable, the reliability of the whole machine would be $2 \times$ $10^{-44}$. Experience with the prototype superconducting magnets suggests that even 99 per cent magnet reliability would be hard to achieve.

The United States has many pressing problems that demand immediate government attention and financial commitment, including an annual budget deficit of $\$ 200,000$ million, an AIDS epidemic, 37 million people without medical insurance and thousands of families who are home- less. There are more urgent needs for $\$ 7,000$ million than a $40-\mathrm{TEV}$ particle accelerator and the search for the elusive Higgs boson.

Department of Radiation Medicine,

ROBERT J. YAES

University of Kentucky,

Chandler Medical Center,

Lexington, Kentucky 40536-6486, USA

\section{Soviet science}

SIR-Nature often publishes material on the shortcomings of the Soviet Union and East European countries in the organization of science ${ }^{1}$, including an interesting Commentary article ${ }^{2}$ on how scientists "striving to be active members of the world community" can be helped. It is very important now that Soviet science is having to be self-supporting. Government subsidies generally go to the central institutes of the USSR Academy of Sciences, but most Soviet scientists are in educational and industrial institutes. Coming as they often do from academic institutes (for various reasons including better opportunities for promotion), the leading scientists there are top quality scientists.

If the goodwill exists, many things could be done:

(1) Talented young scientists could be allowed to compete for grants and postdoctoral and doctoral student positions in Western institutions (they can at present apply only for A. von Humboldt Foundation grants in Germany).

(2) Research grants of international or other organizations could be allocated to Soviet scientists for cooperation in fundamental and applied investigations. Even small projects for chemical reagents and spares would be valuable.

(3) Lecture tours abroad could be of great advantage both for visiting Soviet scientists and for their hosts. Special travel grants could be made.

(4) Many interesting scientific papers are published only in Russian, only part of them being translated, but much later. Leading international journals could accept worthy papers in Russian, organizing translation themselves, their efforts rewarded by novel ideas and techniques. It is appropriate to mention here that Russians made the preliminary investigations leading later to Nobel prize-winning work, namely that of Barnard for heart transplantation (Demikhov) and of Senger and Gilbert for DNA sequencing (Mirzabekov and Sverdlov).

Institute for Biotechnology,

RENAT ZHDANOV*

\section{Moscow 117246, USSR}

* Present address: Department of Clinical Studies, Ontario Veterinary College, University of Guelph, Guelph, Ontario NIG 2W1, Canada.

1. Nature 336, 2 (1988); 341, 563 (1989); 101 (1990). 2. Fisher, G.W., Grew, P.C. \& Yardley, B. Nature 342, 731732 (1989). 